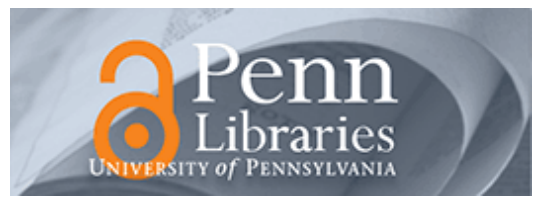

University of Pennsylvania

ScholarlyCommons

Marketing Papers

Wharton Faculty Research

2017

\title{
The Illusion of Multitasking and Its Positive Effect on Performance
}

\author{
Shalena Srna \\ University of Pennsylvania \\ Rom Y. Schrift \\ University of Pennsylvania
}

Gal Zauberman

Follow this and additional works at: https://repository.upenn.edu/marketing_papers

Part of the Behavioral Economics Commons, Cognition and Perception Commons, Cognitive

Psychology Commons, Experimental Analysis of Behavior Commons, Marketing Commons, Performance Management Commons, and the Social Psychology Commons

\section{Recommended Citation}

Srna, S., Schrift, R. Y., \& Zauberman, G. (2017). The Illusion of Multitasking and Its Positive Effect on Performance. Retrieved from https://repository.upenn.edu/marketing_papers/322

This is an unpublished manuscript.

This paper is posted at ScholarlyCommons. https://repository.upenn.edu/marketing_papers/322

For more information, please contact repository@pobox.upenn.edu. 


\title{
The Illusion of Multitasking and Its Positive Effect on Performance
}

\author{
Abstract \\ Multitasking is pervasive. With technological advancements, the desire, ability, and often necessity to \\ engage in multiple activities concurrently are paramount. Although multitasking refers to the \\ simultaneous execution of multiple tasks, most activities that require active attention cannot actually be \\ done simultaneously. Therefore, whether a certain activity is considered multitasking is often a matter of \\ subjective perception. The current paper demonstrates the malleability of what people perceive as \\ multitasking, showing that the same activity may or may not be construed as multitasking. Importantly, \\ although engaging in multiple tasks may diminish performance, we find that, holding the activity constant, \\ the mere perception of multitasking actually improves performance. Across 23 incentive-compatible \\ studies, totaling 6,768 participants, we find that those who perceived an activity as multitasking were \\ more engaged, and consequently outperformed those who perceived that same activity as single-tasking.

\section{Keywords} \\ multitasking, performance, perception, engagement, pupil dilation \\ Disciplines \\ Behavioral Economics | Business | Cognition and Perception | Cognitive Psychology | Experimental \\ Analysis of Behavior | Marketing | Performance Management | Social Psychology

\section{Comments} \\ This is an unpublished manuscript.
}




\title{
The Illusion of Multitasking and Its Positive Effect on Performance
}

\author{
Shalena $\operatorname{Srna}^{1 *}$ \\ Rom Y. Schrift ${ }^{1}$ \\ Gal Zauberman ${ }^{2}$
}

\section{Author Notes:}

${ }^{1}$ Marketing Department, The Wharton School, University of Pennsylvania, 700 Jon M.

Huntsman Hall, University of Pennsylvania, Philadelphia, PA 19104

${ }^{2}$ Marketing Department, Yale School of Management, Yale University, 165 Whitney Avenue, Evans Hall 5532 Yale University New Haven, CT 06511

* Corresponding author: E-mail: ssrna@wharton.upenn.edu; 707-569-6927 


\begin{abstract}
Multitasking is pervasive. With technological advancements, the desire, ability, and often necessity to engage in multiple activities concurrently are paramount. Although multitasking refers to the simultaneous execution of multiple tasks, most activities that require active attention cannot actually be done simultaneously. Therefore, whether a certain activity is considered multitasking is often a matter of subjective perception. The current paper demonstrates the malleability of what people perceive as multitasking, showing that the same activity may or may not be construed as multitasking. Importantly, although engaging in multiple tasks may diminish performance, we find that, holding the activity constant, the mere perception of multitasking actually improves performance. Across 23 incentive-compatible studies, totaling 6,768 participants, we find that those who perceived an activity as multitasking were more engaged, and consequently outperformed those who perceived that same activity as single-tasking.
\end{abstract}

Keywords: multitasking, performance, perception, engagement, pupil dilation 
The Illusion of Multitasking and Its Positive Effect on Performance

In today's technology-dependent world, multitasking is an integral part of daily life (Ophir, Nass, \& Wanerm, 2009; Strayer, Drews, \& Johnston, 2003). People frequently engage in two or more tasks simultaneously like switching between tabs on computers and smartphones, checking email and social media, playing games, and surfing the web. A recent survey of consumers' mobile habits reported that individuals frequently use their smartphones while watching a movie, during a dinner date, and even at church (Jumio, 2013). Multitasking is also prevalent in the workplace, where most environments necessitate working under time pressure on several tasks simultaneously (Gonzalez \& Mark, 2004; Kreckler et al, 2008; O’Conaill \& Frohlich, 1995).

Aside from the prevalence of multitasking, the ability to multitask is also seen as a highly desirable trait (Wang \& Tchernev, 2012). In a survey we conducted with 434 participants (sampled based on age, income, and gender to reflect the US population), we found that $84 \%$ of participants reported that the ability to multitask is an important trait to have and $93 \%$ said they could actually multitask better than or as well as the average person (see Table 1). 
Table 1: Results from the sample broken down by gender, age, and income. Percentages represent the number of participants who selected a certain response within a specific demographic category.

\begin{tabular}{|c|c|c|c|c|}
\hline & \multirow[b]{2}{*}{$\begin{array}{c}\begin{array}{c}\% \text { of } \\
\text { sample }\end{array} \\
\end{array}$} & \multirow{2}{*}{$\begin{array}{c}\text { How capable do you } \\
\text { think you are at } \\
\text { multitasking compared to } \\
\text { an average person? (1 } \\
\text { Much worse, } 4 \text { About the } \\
\text { same, } 7 \text { Much better) } \\
\\
\text { \% chose } \geq 4\end{array}$} & \multirow{2}{*}{$\begin{array}{l}\text { To what extent do you } \\
\text { believe that the ability to } \\
\text { multitask is an important } \\
\text { trait to have? (1 Not at } \\
\text { all important, } 4 \text { Neither } \\
\text { important nor } \\
\text { unimportant, } 7 \\
\text { Extremely important) } \\
\text { \% chose }>\mathbf{4}\end{array}$} & $\begin{array}{l}\text { In your day-to-day life, } \\
\text { on average, how } \\
\text { frequently do you } \\
\text { multitask? (1 Never, } 4 \\
\text { Sometimes, } 7 \text { Always) }\end{array}$ \\
\hline & & & & $\%$ chose 6 or 7 \\
\hline Overall & $100 \%$ & $93.32 \%$ & $84.10 \%$ & $47.00 \%$ \\
\hline \multicolumn{5}{|l|}{ Gender } \\
\hline male & $49.77 \%$ & $92.59 \%$ & $80.09 \%$ & $39.35 \%$ \\
\hline female & $50.23 \%$ & $94.04 \%$ & $88.07 \%$ & $54.59 \%$ \\
\hline \multicolumn{5}{|l|}{ Age } \\
\hline $18-24$ years $^{1}$ & $4.84 \%$ & $95.24 \%$ & $100.00 \%$ & $61.90 \%$ \\
\hline $25-34$ years & $20.51 \%$ & $94.38 \%$ & $89.89 \%$ & $60.67 \%$ \\
\hline $35-44$ years & $18.20 \%$ & $97.47 \%$ & $93.67 \%$ & $60.76 \%$ \\
\hline $45-54$ years & $20.05 \%$ & $93.10 \%$ & $82.76 \%$ & $49.43 \%$ \\
\hline 55-64 years & $16.82 \%$ & $95.89 \%$ & $80.82 \%$ & $35.62 \%$ \\
\hline $65+$ years & $19.59 \%$ & $85.88 \%$ & $69.41 \%$ & $23.53 \%$ \\
\hline \multicolumn{5}{|l|}{ Income } \\
\hline under $\$ 15 \mathrm{k}$ & $12.21 \%$ & $92.45 \%$ & $83.02 \%$ & $33.96 \%$ \\
\hline$\$ 15 \mathrm{k}-\$ 25 \mathrm{k}$ & $11.29 \%$ & $87.76 \%$ & $81.63 \%$ & $55.10 \%$ \\
\hline$\$ 25 \mathrm{k}-\$ 35 \mathrm{k}$ & $10.60 \%$ & $93.48 \%$ & $91.30 \%$ & $41.30 \%$ \\
\hline$\$ 35 \mathrm{k}-\$ 50 \mathrm{k}$ & $13.13 \%$ & $92.98 \%$ & $85.96 \%$ & $45.61 \%$ \\
\hline$\$ 50 \mathrm{k}-\$ 75 \mathrm{k}$ & $22.58 \%$ & $91.84 \%$ & $78.57 \%$ & $46.94 \%$ \\
\hline$\$ 75 \mathrm{k}-\$ 100 \mathrm{k}$ & $11.52 \%$ & $98.00 \%$ & $84.00 \%$ & $58.00 \%$ \\
\hline$\$ 100 \mathrm{k}-\$ 150 \mathrm{k}$ & $11.75 \%$ & $96.08 \%$ & $84.31 \%$ & $47.06 \%$ \\
\hline$\$ 150 \mathrm{k}+$ & $6.91 \%$ & $96.67 \%$ & $93.33 \%$ & $50.00 \%$ \\
\hline
\end{tabular}

Early research on multitasking examined how working concurrently on two or more tasks affects human performance on various activities (Borger, 1963; Creamer, 1963). This research found that when working on multiple non-automatic tasks individuals cannot actually perform the tasks simultaneously but rather alternate between different activities, engaging only in a single task at any given time (Kieras et al, 2000; Pashler, 1994). Studies have shown such

\footnotetext{
${ }^{1}$ Although $11 \%$ of the US population is between 18 and 24 years old, due to sampling error, this group is underrepresented in our sample.
} 
switching behavior is detrimental to performance due to cognitive-processing limitations and residual attention on the previous task (e.g. Leroy, 2009; Levy \& Pashler, 2001; Pashler, 1994).

Even when people feel they are multitasking, they process only a single task at a time. Therefore, first, this paper postulates that multitasking is often merely a matter of subjective perception. That is, holding the actual activity constant, some situations may cause people to perceive their overall activity as multitasking, whereas other situations may cause people to construe the same activity as single-tasking. Second, we propose that the way people mentally construe an activity, either as multitasking or single-tasking, can affect their performance on that activity. For example, imagine you are asked to watch a video and type everything that is said during the video. If you construe this activity as transcribing, you will probably consider this a single task. However, if you construe this activity as including two distinct tasks done simultaneously, watching the video and typing, you will more likely consider this activity as multitasking. The central question of this paper is how the difference in perception of the same activity might impact performance.

It is important to investigate the malleability of multitasking perception and its impact on performance because almost any "single" activity could be broken down into its components and construed as multitasking. Inversely, multiple distinct activities can be framed as a single task. Thus, studying the malleability of people's multitasking perceptions and how they affect performance has important implications across many domains.

The current paper not only studies the malleability of peoples' multitasking perceptions, but also examines how such perceptions may affect people's performance on the activity. As alluded to earlier, the ability to multitask well is considered a desirable trait. We propose that this desire may motivate individuals to become more engaged in an activity when it is perceived as a 
multitasking activity. This engagement, we hypothesize, could positively influence performance. To the best of our knowledge, the current paper is the first to explore how merely framing a given activity as either single-tasking or multitasking affects behavior.

Across 23 incentive-compatible studies with 6,768 participants who engaged in different tasks (e.g., transcribing, taking a virtual tour in an art gallery, and solving different puzzles and anagrams), we consistently find that the mere perception of multitasking improves performance on the activity. Using physiological measures, we find that one key driver for this improvement is greater attention to and engagement with the task.

The current paper's findings do not contradict the extensive research demonstrating the detrimental effects of multitasking, but rather complement it. In particular, previous research on multitasking demonstrated that when asked to work on more than one task, individuals tend to perform worse. However, we focus on the mere perception of multitasking, holding constant the actual activities people engage in. Our research suggests that making people think that they are multitasking as opposed to single-tasking is beneficial to performance. We next report five studies that test the aforementioned hypothesis and conclude with a meta-analysis of all 23 studies we conducted.

\section{Studies 1a and 1b: Multitasking Perceptions and their Impact on Performance}

The goal of Studies $1 \mathrm{a}$ and $1 \mathrm{~b}$ was to test whether framing a certain activity as multitasking, as opposed to single-tasking, improves performance. In Study 1a we asked participants to watch and transcribe an educational video. In Study $1 \mathrm{~b}$ participants took a virtual tour of an art gallery and then were asked questions about its content. In both studies, employing 
incentive-compatible designs, we framed an activity as either multitasking or single-tasking and examined how such framing impacted performance.

\section{Study 1a: Method}

One hundred and sixty-two participants $(62 \%$ female; mean age $=21.04)$ were recruited from a northeastern university to participate in a lab session in exchange for payment. Sample size (for this study and all reported studies) was determined in advance based on effect sizes from initial studies and the number of participants we could recruit. All participants were asked to watch an educational video clip and transcribe what was said in the clip. Then, participants were randomly assigned to either the multitasking or single-tasking conditions.

Participants assigned to the multitasking condition were told that they would be working on two tasks concurrently and, therefore, would need to multitask. The first task, entitled the Learning Task, was described as a test of individuals' learning abilities and required participants to watch an educational video from Animal Planet's Shark Week. The second task, entitled the Transcribing Task, was described as a test of individuals' writing skills that required participants to transcribe exactly what was said in the video. Thus, through framing, we intended to make participants in this condition feel as if they were working on two separate tasks concurrently.

Participants assigned to the single-tasking condition were asked to perform the exact same activity that was merely framed differently. Specifically, participants in this condition were told that they would be working on a Learning Task meant to test individuals' learning and writing abilities. The task was described as watching and transcribing an educational video from Animal Planet's Shark Week. Thus, in both conditions participants performed the exact same activity and were told that both their learning and writing abilities would be tested. However, in 
the single-tasking condition the activity was framed as a single task and in the multitasking condition it was framed as two tasks done concurrently. In a separate pretest using the same population from a northeastern university we validated that this framing manipulation worked as intended (see supplemental materials for details).

All participants earned an additional $\$ 0.02$ for each word they correctly transcribed and could work for as long as they liked up until the video ended after 6 minutes. Thus, our first measure of performance was how many words participants transcribed. The second measure of performance tested participants' comprehension of the information provided in the video. Specifically, at the end of the study we administered a 10-question multiple-choice pop quiz with questions about the video's content.

\section{Results}

The first measure of performance we analyzed was the number of words transcribed in each condition. Participants assigned to the multitasking condition transcribed significantly more words $(M=274.13, \mathrm{SD}=126.24)$ than participants assigned to the single-tasking condition $(M=$ 229.60, $\left.\mathrm{SD}=137.22 ; F(1,160)=4.63, p=.033, \eta^{2}=.028\right)$.

As an additional measure of performance we checked how well participants performed on the pop quiz. As predicted, participants assigned to the multitasking condition performed significantly better on the quiz $(M=6.60, \mathrm{SD}=1.80)$ than participants assigned to the singletasking condition $\left(M=5.81, \mathrm{SD}=2.31, F(1,160)=5.82, p=.017, \eta^{2}=.035\right)$.

We next analyzed how long participants spent transcribing the video by first logtransforming time spent on the task. Since the video ended for all participants after six minutes, the data were right-censored. Thus, we used a cox regression survival analysis to find that there 
was not a significant difference in persistence $\left(T_{\log \text { time }}\right.$ multitasking $=2.43, \mathrm{SD}=0.23 ; T_{\text {log time single- }}$ tasking $\left.=2.32, \mathrm{SD}=0.35 ; \mathrm{Wald}-\chi^{2}(1, \mathrm{~N}=162)=2.15, p=.142\right)$. This analysis method is employed on all subsequent persistence analyses.

Moreover, even when controlling for time differences, participants assigned to the multitasking condition outperformed those assigned to the single-tasking condition. Specifically, participants in the multitasking condition wrote more words per second $(M=0.91, \mathrm{SD}=0.24)$ than participants assigned to the single-tasking condition $(M=0.84, \mathrm{SD}=0.28 ; F(1,160)=3.49$, $\left.p=.064, \eta^{2}=.021\right)$

\section{Study 1b: Method}

One hundred ten participants $(61 \%$ female; mean age $=22.84)$ were recruited from a behavioral lab at a northeastern university to take part in an hour-long lab session. All participants took the same virtual tour of an art gallery with three exhibits displaying different paintings and sculptures by various artists. An auditory narrative that described the history and style of the art pieces accompanied the virtual tour (stimuli adapted from Barasch, Diehl, Silverman, \& Zauberman, 2016). Prior to the beginning of the tour, we randomly assigned participants to one of two conditions: multitasking or single-tasking.

Participants assigned to the multitasking condition were told that the tour included a visual component (exhibits, paintings, etc.) and an auditory component (narration about the art and their history). They were also instructed that many people do not realize that watching a tour involves multitasking between observing the visual component and listening to the auditory component.

Participants assigned to the single-tasking condition were given the same instructions and 
were also told that the tour included a visual component (exhibits, paintings, etc.) and an auditory component (narration about the art and their history). Although participants were reminded that they would observe the visual component and listen to the auditory component, they were not told that this activity involved multitasking. Participants in both conditions were instructed they could quit the tour at any point in time. In a separate pretest using the same population from a northeastern university, we validated that the framing manipulation worked as intended (see supplemental materials for details).

Following the tour, participants responded to twenty-nine questions about the information they learned during the tour. The test involved questions relating to both components of the tour (e.g., identifying the artwork they saw and recalling verbal information the narrator mentioned). Finally, participants indicated how enjoyable and how boring they found the virtual tour to be (each measured on a 1 to 7 scale).

\section{Results}

As predicted, participants who perceived their activity as multitasking performed significantly better on the test and answered more questions correctly $(M=21.00, \mathrm{SD}=5.29)$ compared to participants assigned to the single-tasking condition $(M=18.23, \mathrm{SD}=5.71$; $\left.F(1,108)=6.95, p=.010, \eta^{2}=.060\right)$. Analyzing the auditory and visual questions separately produced the same pattern (Auditory questions: $M_{\text {multitasking }}=9.91, \mathrm{SD}=2.39, M_{\text {single-tasking }}=8.61$, $\mathrm{SD}=2.79 ; F(1,108)=6.86, p=.010, \eta^{2}=.060 ;$ Visual questions: $M_{\text {multitasking }}=11.09, \mathrm{SD}=3.51$, $\left.M_{\text {single-tasking }}=9.63, \mathrm{SD}=3.58 ; F(1,108)=4.72, p=.032, \eta^{2}=.042\right)$.

An analysis of how long participants worked on the task in each condition (data were right-censored at 5 minutes and 18 seconds) did not reveal a significant difference $\left(T_{\log }\right.$ time 
multitasking $=2.40, \mathrm{SD}=0.29 ; T_{\text {log time single-tasking }}=2.33, \mathrm{SD}=0.39 ; \mathrm{Wald}-\chi^{2}{ }_{(1, \mathrm{~N}=110)}=0.69, p$

$=.406)$.

Although we did not find evidence that participants in the multitasking condition enjoyed the assignment more $(F(1,108)=2.04, p=.156)$, they did report being significantly less bored during the tour $(M=3.87, \mathrm{SD}=1.75)$ than participants in the single-tasking condition $(M=4.71$, $\left.\mathrm{SD}=1.65 ; F(1,108)=6.79, p=.010, \eta^{2}=.059\right)$.

\section{Discussion}

These two studies demonstrate that framing an activity as a multitasking activity can benefit performance. Study $1 \mathrm{~b}$ further demonstrated that the perception of multitasking is associated with reduced boredom. This indicates that the improved performance in the multitasking condition may be a result of increased engagement with the task. We test this more directly using physiological measures in Study 3.

Admittedly, in these studies we triggered the perception of multitasking by explicitly telling participants that the activity involved multitasking. Accordingly, one concern may be that the effect is driven by mentioning the notion of multitasking and not by how individuals spontaneously construe their activity. Although this is not concerning from an applied perspective (i.e., using an explicit manipulation to trigger multitasking perceptions is easy to implement in many contexts), it is important to examine whether the effect persists without explicitly mentioning multitasking. We address this in Study 2.

\section{Studies 2a and 2b: Measuring and Manipulating the Perception of Multitasking and Its Impact on Performance}


In this study participants were asked to complete an incentive-compatible task that involved solving two types of puzzles. To address the role of explicitly instructing participants to multitask, in Study 2a we merely measured (as opposed to manipulated) whether participants construed their activity as either multitasking or single-tasking. Using this natural variation in how people construed the activity, we examined how such perceptions were correlated with their performance on the tasks. To support causal claims, in Study $2 \mathrm{~b}$ we used the same materials but manipulated multitasking perceptions instead of measuring them.

\section{Study 2a: Method}

Eighty participants $(43 \%$ female; mean age $=36.66)$ were recruited from Amazon’s Mechanical Turk to take part in this study ${ }^{2}$. Participants were asked to work on an incentivecompatible assignment comprised of two distinct puzzles. The first puzzle was a word-puzzle in which participants observed a 15 by 15 matrix of letters and were asked to find as many words as possible in a horizontal, vertical, or diagonal pattern in the matrix. The second puzzle was an anagram task in which participants observed a 10-letter string and were asked to come up with as many words as possible using the letters in the string. Each correct answer (i.e., a 4 or more letters-long word) earned participants an additional \$0.01. The two puzzles appeared on the same screen side by side, and participants worked on the tasks concurrently for four minutes and could submit as many words they could find (See Figure 1).

\footnotetext{
${ }^{2}$ One participant was more than two standard deviations from the mean performance and was therefore excluded from the analyses. Results hold if this participant is included.
} 
Fig. 1: Puzzles used in Study 2

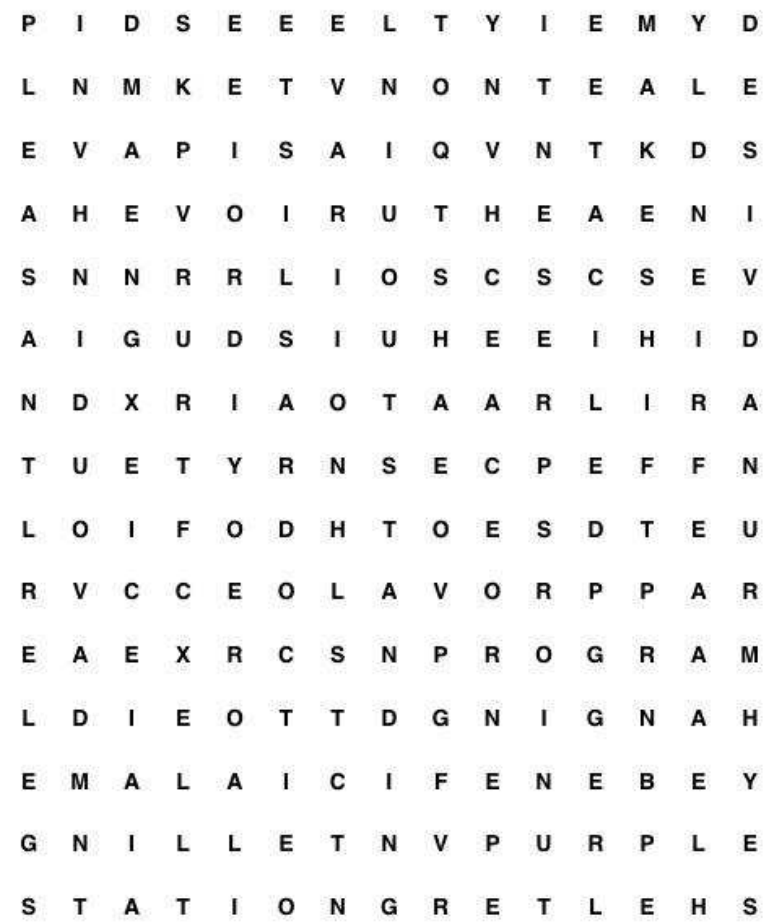

\section{EMTTECSOWF}

After participants finished working on the task, we measured the extent to which they perceived their activity as either multitasking or single-tasking using two types of measurements. First, to prevent participants from stating post hoc that they perceived their activity as multitasking, and to increase the validity of their responses, we applied an incentive-compatible response format. Specifically, after participants finished working on the task we told them that they would be matched with a partner, and that they and their partner would need to indicate whether they perceived working on the tasks as a multitasking or single-task activity. If their partner and they both responded to the question in the same way, they would each receive an 
additional bonus payment. Four additional binary items were used to measure perceptions of multitasking (see supplemental materials for details). Responses to all five binary measures were combined into a single measure of multitasking perception.

\section{Results}

We regressed the number of words found in the puzzles on the measure of multitasking perception and found a significant positive relationship between the perception of multitasking and performance $(\beta=4.33, t=2.47, p=.016)$. That is, we find that the more participants felt they were multitasking the better they performed. Obviously, one cannot make any causal claims based on this study alone because reverse causality could drive the association. To further test causality, in Study $2 \mathrm{~b}$ we use the exact same activity but instead of measuring the perception of multitasking we manipulate it.

\section{Study 2b: Method}

Two hundred and thirty-seven paid online participants were recruited from Amazon.com's Mechanical Turk $(55 \%$ female; mean age $=36.33)$. Participants were asked to work on an incentive-compatible assignment comprised of two distinct puzzles as described in Study 2a. Participants were randomly assigned to one of two framing manipulations. In the multitasking condition, the two tasks were described as relating to two different studies ("Perceptual Study" and "Identification Study"), were separated on the screen by a vertical line, and had a different background color. The single-tasking condition described both tasks as being part of the same study ("Perceptual-Identification Study"), and were not distinguished by different background colors or separated by a line. Unlike Study 2a, participants were allowed to 
quit the tasks at any time up until four minutes, thus enabling us to examine persistence as another indication of performance.

Since the framing manipulation was relatively subtle, we also included an additional factor intended to further strengthen the manipulation. In particular, we also manipulated (between-subjects) whether or not we disclosed to participants that in this study some participants would work on a single study while others would work on two studies at the same time. The disclosure manipulation did not produce any main effects or interactions on any of the dependent variables or with the framing manipulation. We therefore collapsed the analyses and do not discuss this factor further. This does not substantially change the pattern of results.

As a manipulation check, participants indicated to what extent they felt they were multitasking $(1=$ Not at all, $4=$ Somewhat, $7=$ Totally $)$ and whether they felt like they were completing two different tasks, a single task with two components, or a single task (selected one of the three descriptions that matched their experience).

For exploratory purposes, upon finishing their assignment, participants in all conditions were asked to answer several questions about their multitasking habits and feelings of productivity (reported in the supplemental materials). None of these measures moderated the effect; therefore, we do not discuss these further.

\section{Results}

Manipulation checks. Participants assigned to the multitasking condition indicated that they perceived their activity as multitasking $(M=4.40, \mathrm{SD}=1.78)$ to a greater extent than those assigned to the single-tasking condition $(M=2.52, \mathrm{SD}=1.53 ; F(1,235)=76.06, p<.001)$. Furthermore, of the participants who were assigned to the multitasking [single-tasking] 
condition, $46 \%$ [5\%] indicated perceiving their activity as working on two separate tasks $\left(\right.$ Pearson- $\left.\chi_{(1, \mathrm{~N}=237)}^{2}=51.35, p<.001\right)$, and $11 \%[71 \%]$ indicated perceiving their activity as working on a single task (Pearson- $\left.\chi_{(1, \mathrm{~N}=237)}^{2}=88.81 p<.001\right)$. Thus, the manipulation worked as intended.

Performance. An ANOVA revealed that participants assigned to the multitasking condition submitted, on average, more words $(M=14.42, \mathrm{SD}=7.53)$ than those assigned to the single-tasking condition $\left(M=8.08, \mathrm{SD}=5.03 ; F(1,235)=57.86, p<.001, \eta^{2}=.198\right)^{3}$. This improvement in performance resulted in an average increase of $78 \%$ in the bonus payment for those who perceived they were multitasking.

Persistence. Participants worked on the puzzles for at most four minutes, but could quit at any point prior to that. We analyzed how long participants worked on the task and found that participants who were assigned to the multitasking condition persisted longer $(M=2.35, \mathrm{SD}=$ $0.14)$ than those assigned to the single-tasking condition $\left(M=2.26, \mathrm{SD}=0.27 ;\right.$ Wald- $\chi^{2}(1, \mathrm{~N}=237)$ $=14.02, p<.001)$.

Moreover, even after controlling for the time participants spent on the tasks, participants in the multitasking condition still performed better $(F(1,234)=46.49, p<.001)$, suggesting the quality of work, and not only the overall time spent on the task (i.e., persistence), drove the improvement in performance.

\section{Discussion}

By both manipulating and measuring multitasking perceptions, the results of Studies $2 \mathrm{a}$ and $2 \mathrm{~b}$ add to those of Study 1 to support the notion that performance on a given activity

\footnotetext{
${ }^{3}$ Results hold after adjusting for unequal variance across conditions. See Table S1 in supplemental materials for all performance mean, median, and standard deviations for each study.
} 
improves when individuals construe their activity as multitasking. Across different incentivecompatible activities, participants performed better and earned more when the same activity was merely construed as multitasking. In Study 3, employing the same paradigm as Study 2b, we use eye-tracking measures and pupil dilation to examine the individual-specific pattern of switches between tasks and whether the improvement in performance is indeed triggered by greater engagement with the task.

\section{Study 3: Physiological Measures of Engagement}

In this study, we tracked participants' eye movements and pupil dilation while working on the tasks. This methodology enabled us to examine participants' switching patterns and also their engagement using a validated physiological measure (e.g., Beatty \& Lucero-Wagoner, 2000; Hoeks \& Levelt, 1993; Kahneman, 1973).

\section{Method}

One hundred and fifteen participants were recruited from a behavioral lab at a northeastern university $(60 \%$ female; mean age $=20.46)$. The procedure was identical to that employed in Study 2b, but while participants worked on the tasks, we used SMI RED-m eyetracking equipment to track their eye movements and pupil dilation.

To ensure that the use of different background colors did not impact the pupil dilation measures, we counterbalanced all background colors across conditions. Further, we verified that the level of luminance across conditions (which may affect pupil dilation) was nearly identical 
by calculating the mean luminance over the pixels ${ }^{4}$ and scaling them from $0-255$, (since the colors were counterbalanced there were two task stimuli per condition: Single task $=194.362$, 194.443 vs. Multitask $=194.586,194.634)$ and thus unlikely to have caused a change in pupil dilation.

\section{Results}

Consistent with eye-tracking research practices, we made several exclusions based on data quality and criteria determined a priori. Participants were excluded if (i) their time on the task exceeded two standard deviations from the average time (five participants; results hold if not excluded), (ii) if the eye-tracking device did not read their pupil dilation (2 participants), or (iii) if they had other technical difficulties (1 participant), leaving us with a total of 107 participants.

Performance. As predicted participants identified more words in the multitasking condition $(M=18.21, \mathrm{SD}=11.93)$ than in the single-tasking condition $(M=10.65, \mathrm{SD}=7.94$; $F(1,105)=13.84, p<.001)$.

Persistence. We found that most participants worked for the full 4 minutes (potentially because of the salient presence of a research assistant who monitored the eye-tracking equipment). Thus, the framing manipulation had no significant effect on how long participants worked on the task $(p>.25)$.

Pupil dilation. Participants' average pupil dilation was larger in the multitasking condition $(M=3.90, \mathrm{SD}=0.51)$ than in the single-tasking condition $(M=3.64, \mathrm{SD}=0.49$; $F(1,105)=7.12, p=.009)$. These results hold when analyzing either median or maximum pupil dilation (see supplemental materials). The effect of the multitasking framing on pupil dilation

\footnotetext{
${ }^{4}$ We use a Gamma correction to account for nonlinearity in processing luminance (Poynton, 2002)
} 
remained significant even after controlling for the number of switches participants made $(F(1$, $99)=5.90, p=.017)^{5}$. A mediation analysis using a bootstrap estimation approach with 5,000 samples (model 4 from the PROCESS macro; Hayes, 2013) supported the assertion that the increase in pupil dilation mediated the impact of task framing on performance $(\beta=0.59, \mathrm{SE}$ $=.34,95 \% \mathrm{CI}=.1008,1.5524)$. Figure 2 depicts participants' average pupil dilation over time across conditions.

Fig. 2: Average pupil dilation in the multitasking and single-tasking conditions in Study 3.

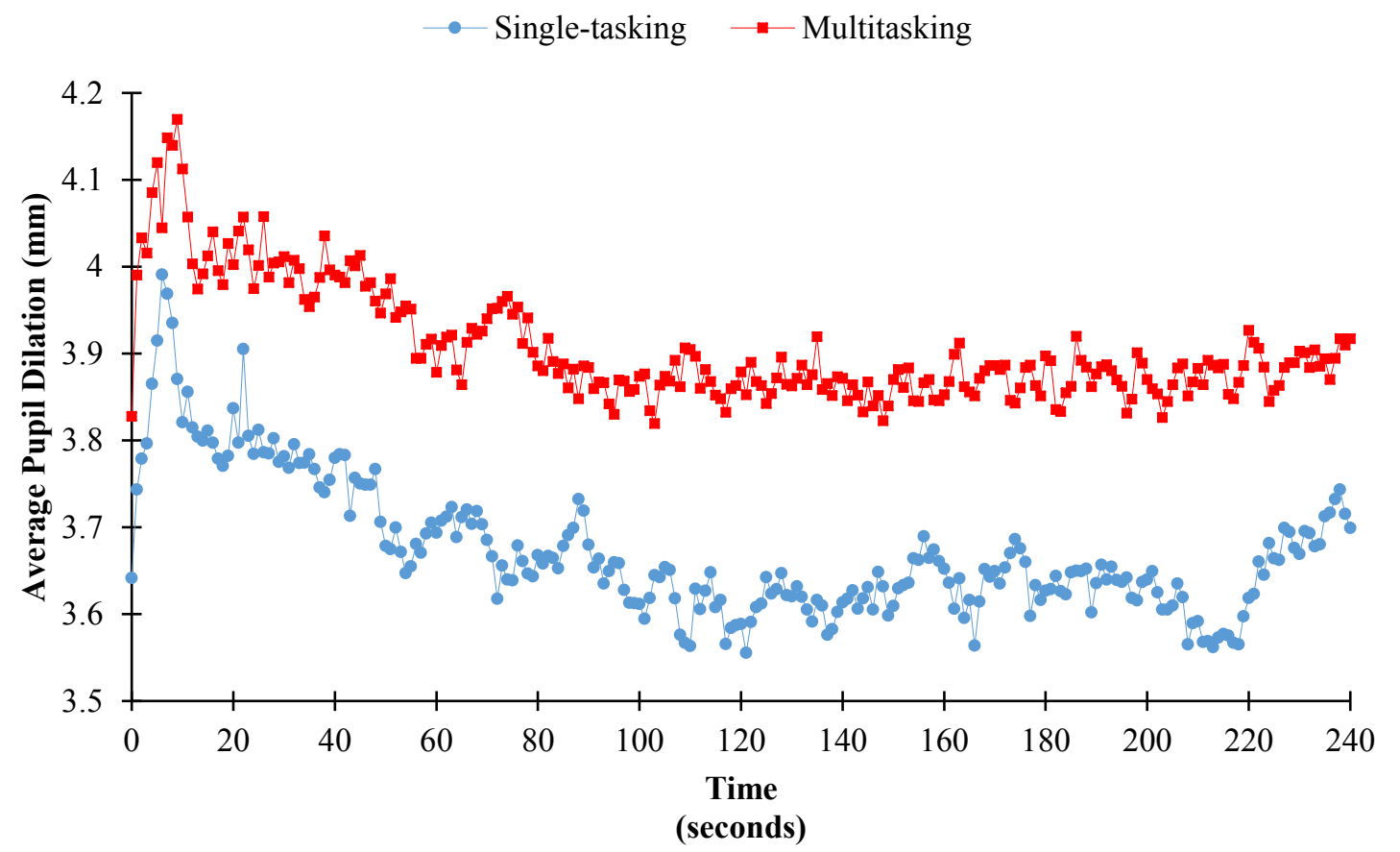

Given the correlational nature of any mediation analysis, one should be cautious with causality interpretations. For example, although manipulating the perception of multitasking increased performance (i.e., participants found more words), reverse causality between pupil

${ }^{5}$ The sample size changed for this analysis because the eye-tracking equipment failed to record the exact fixation location of five participants, thus we could not calculate their number of switches. 
dilation and performance needs to be considered. In particular, participants' pupils might have been dilated due to happiness and excitement from finding more words. Although we cannot fully rule out this account, an additional analysis that controls for the number of words found in each condition casts doubt on this interpretation. Specifically, when analyzing average pupil dilation up until participants found their first word in the puzzles, participants in the multitasking condition still had a greater average pupil dilation $(M=3.93, \mathrm{SD}=0.52)$ than those in the singletasking condition $(M=3.71, \mathrm{SD}=0.57 ; F(1,105)=4.28, p=.041)$. Similar patterns were found when examining pupil dilation up until the second, third, fourth, and fifth word found. Thus, regardless of the number of words found in the puzzles, participants in the multitasking condition exhibited greater physiological signs of engagement than participants in the single-tasking condition.

\section{Replications and Meta-Analysis}

Using the same paradigm we used in Studies 2 and 3, we replicated the pattern of results across additional studies exploring different facets of this effect ( 4 of these are reported in the supplemental materials). Across these studies, participants in the multitasking condition performed significantly better than participants in the single-tasking condition. We found the increase in performance occurred regardless of whether participants had a sense of agency over the decision to multitask (Study 4), and regardless of their level of extraversion and neuroticism (Study 5). Further, the perception of multitasking did not change participants' locus of control or heighten their self-efficacy (Study 6). Finally, we did not find consistent evidence supporting the notion that a shift in mindset drove the effect (Study 7; i.e., a productivity mindset, Keinan \& Kivetz, 2011). 
It is important to note that, while we kept the actual activity fixed across the both conditions in Studies 2-6, we did not control or restrict participants' work sequence. That is, some participants may have switched more often than others and such variation in work sequence may have partially driven the results. Although such account cannot fully explain the results obtained in Studies 1a and 1b, we conducted an additional study that fully controlled the work sequence (see Study 7 in the supplemental materials). In this study, using a different paradigm, we externally forced a specific pattern by which participants switched back and forth between the tasks. We found that even after controlling their switching patterns, participants who perceived their activity as multitasking persisted longer and answered more questions correctly.

To assess the size and robustness of the focal effect, we computed a meta-analysis of all 23 studies (6,768 participants), using different study paradigms. This analysis finds a robust medium size effect (Cohen's $d=.501,95 \%$ Confidence Interval $.416, .586$; Wilson, 2006) with an average increase of $42 \%$ in the task-based payment. Additionally, pooling only the results from the 12 studies $(\mathrm{N}=3,527)$ in which we explicitly measured participants' perceptions of multitasking versus single-tasking, we find a small positive and significant relationship between perceptions of multitasking and performance (using z-scores; $\beta=0.06, t=3.67, p<.001$ ) ${ }^{6}$

\section{General Discussion}

Multitasking is often a matter of perception. Although the term refers to the concurrent execution of multiple tasks, most tasks that require active attention cannot be done simultaneously. In the current paper, we demonstrate the malleability of people's perceptions of multitasking by showing that the same activity may or may not be perceived as multitasking.

\footnotetext{
${ }^{6}$ Note that due to measurement error, this correlation, while significant, is weaker that the effect of manipulations as captured by the meta-analysis.
} 
Further, by both manipulating and measuring multitasking perceptions, we find that holding the activity constant, the mere perception of multitasking improves performance. A mediation analysis supports that heightened engagement is an important driver of this effect.

It is important to note that our findings do not suggest that multitasking is superior to single-tasking. Voluminous research demonstrates that working on more than one task is detrimental to performance. However, we argue and consistently demonstrate that, holding the $\operatorname{task}(\mathrm{s})$ constant, the mere perception of multitasking is beneficial to performance. Stated differently, one implication of this research is that breaking down a single task into its separate components and merely creating the illusion of multitasking could improve peoples' performance on this task. For example, if we were to mention that reading this article entails two distinct tasks (e.g., switching back and forth between the text and figures), to the extent that this framing would trigger a perception of multitasking, one should observe an improvement in performance (i.e., better comprehension). Furthermore, the findings suggest that if people are already doing multiple tasks, making them aware that this activity is multitasking should increase engagement and help them perform better. So if you are doing other activities while reading this article, such as answering urgent emails, realizing you are multitasking should improve your engagement and performance in each of these activities.

Why is this happening? We find across multiple studies, using both self-reported and physiological measures, that the mere perception of multitasking increases engagement in the activity. We conjecture that one reason for this increase in engagement is the motivation to appear as an adept multitasker. This possibility seems particularly plausible in light of the findings that multitasking is a desirable trait; however, other reasons may underlie this boost in engagement. For example, individuals might perceive multitasking as more challenging and 
therefore focus more on the work. Furthermore, it is also plausible that, by making the task's components more salient in the multitasking condition, we added more structure to the activity, which helped participants perform better. According to this account, one would expect that individuals with high need for structure would benefit more from the multitasking framing manipulation. However, we found in Study 1a that participants' need for structure (Thompson et al, 2001) did not moderate the observed effect (see supplemental materials for the detailed analysis). Additional research is needed to more conclusively test these accounts and other psychological mechanisms driving this robust effect.

In sum, although the prevalence of technology is bringing multitasking to almost every aspect of life, social scientists have so far focused on the detrimental effects of doing multiple tasks rather than focusing on a single task. By contrast, we make a different comparison: given that many activities consist of different components, we examined whether the perception of engaging in multi- or single-tasking impacts performance. We show that in this context, multitasking is a malleable perception that, on its own, benefits rather than harms performance.

\section{Author Contributions}

S. Srna performed the data collection and analysis. All authors jointly developed the studies' concepts and designs and approved the final version of the manuscript.

\section{Declaration of Conflicting Interests:}

The authors declared that they had no conflicts of interest with respect to their authorship or the publication of this article. 


\section{Acknowledgments}

This work was supported by the Wharton Risk Center, Ackoff Doctoral Student Fellowship, and the Wharton Behavioral Lab. 


\section{References}

Barasch, A., Diehl, K., Silverman, J., \& Zauberman, G. (2016). Photographic Memory: The Effects of Volitional Photo-Taking on Memory for Visual and Auditory Aspects of an Experience. Working paper.

Beatty, J. \& Lucero-Wagoner, B. (2000). The Pupillary System. Handbook of psychophysiology, $2,142-162$.

Borger, R. (1963). The Refractory Period and Serial Choice-reactions. Quarterly Journal of Experimental Psychology, 15(1), 1-12.

Creamer, L.R. (1963). Event uncertainty, psychological refractory period, and human data processing. Journal of Experimental Psychology, 66(2), 187.

González, V.M., \& Mark, G. (2004). Constant, Constant, Multi-tasking Craziness: Managing multiple working spheres. In Proceedings of the SIGCHI conference on Human factors in computing systems, 113-20.

Hayes, A.F. (2013). Introduction to Mediation, Moderation, and Conditional Process Analysis. New York, NY: Guilford.

Hoeks, B. \& Levelt, W.J. (1993). Pupillary Dilation as a Measure of Attention: A Quantitative System Analysis. Behavior Research Methods, Instruments, \& Computers, 25(1), 16-26.

Jumio (2013). Consumer Habit Survey. Available at https://www.jumio.com/blog. Retrieved September 6, 2015.

Kahneman, D. (1973). Attention and Effort. Englewood Cliffs, NJ: Prentice-Hall, 18-27.

Keinan, A. \& Kivetz, R. (2011). Productivity Orientation and the Consumption of Collectable Experiences. Journal of Consumer Research, 37(6), 935-50.

Kieras, D., Meyer, D., Ballas, J., \& Lauber, E. (2000). Modern Computation Perspectives on 
Executive Mental Processes and Cognitive Control: Where to from here? Control of Cognitive Processes. Attention and Performance, 12.

Kreckler, S., Catchpole, K., Bottomley, M., Handa, A., \& McCulloch, P. (2008). Interruptions During Drug Rounds: An Observational Study. British Journal of Nursing, 17(21), 132630.

Leroy, S. (2009). Why Is It So Hard To Do My Work? The Challenge of Attention Residue When Switching Between Work Tasks. Organizational Behavior and Human Decision Processes, 109(2), 168-81.

Levy, J. \& Pashler, H. (2001). Is Dual-Task Slowing Instruction Dependent? Journal of Experimental Psychology: Human Perception and Performance, 27(4), 862-69.

Miller, E.K., \& Buschman, T.J. (2015). Working Memory Capacity: Limits on the Bandwidth of Cognition. Daedalus, The American Journal of Arts \& Sciences, 144(1), 112-22.

O'Conaill, B. \& Frohlich, D. (1995). Timespace in the workplace: Dealing with interruptions. In Conference companion on Human factors in computing systems, 262-263.

Ophir, E., Nass, C., \& Wagner, A.D. (2009). Cognitive control in media multitaskers. Proceedings of the National Academy of Sciences USA, 106(37), 15583-15587.

Pashler, H. (1994). Dual-Task Interference in Simple Tasks: Data and Theory. Psychological Bulletin, 116(2), 220-44.

Poynton, C. (2002). Gamma FAQ - Frequently Asked Questions about Gamma. Available at http://www.poynton.com/notes/colour_and_gamma/GammaFAQ.html. Retrieved December 21, 2016.

Strayer, D.L., Drews, F.A., \& Johnston, W.A. (2003). Cell Phone-Induced Failures of Visual Attention During Simulated Driving. Journal of experimental psychology: Applied, 9(1), 
23-32.

Thompson, M.M., Naccarato, M.E., Parker, K.C., \& Moskowitz, G.B. (2001). The Personal Need for Structure and Personal Fear of Invalidity Measures: Historical Perspectives, Current Applications, and Future Directions. In Cognitive social psychology: The Princeton symposium on the legacy and future of social cognition, 19-39.

Tombu, M.N., Asplund, C.L., Dux, P.E., Godwin, D., Martin, J.W., \& Marois, R. (2011). A Unified Attentional Bottleneck in the Human Brain. Proceedings National Academy of Sciences USA, 108(33),13426-31.

Wang, Z. \& Tchernev, J.M. (2012). The "Myth" of Media Multitasking : Reciprocal Dynamics of Media Multitasking, Personal Needs, and Gratifications. Journal of Communication, $62,493-513$.

Wilson, D. B. (2006). Meta-analysis Macros for SAS, SPSS, and Stata. Available at http://mason.gmu.edu/ dwilsonb/ma.html. Retrieved November 6, 2016.

Witt, B.I. \& Lambert, W. (1965). Systems Reference Library IBM Operating System / 360 Concepts and Facilities. IBM Systems Reference Library, 1-91. 
Supplemental Materials

\section{The Illusion of Multitasking and Its Positive Effect on Performance}

Shalena Srna,* Rom Y. Schrift, Gal Zauberman

* Correspondence to: ssrna@,wharton.upenn.edu 


\section{Study 1a}

Manipulation check. We validated our manipulation in a separate pre-test. Using the same population, one hundred and seventy-eight participants were randomly assigned to either read the instructions from the multitasking or the single-tasking condition. First, participants responded whether they would feel as if they were engaging in a single-task or multitask activity. Then, participants answered three more manipulation checks in which they indicated (i) to what extent they would feel they were multitasking ( $1=$ Not at all, $4=$ Somewhat, $7=$ Totally), (ii) the extent to which they would perceive their activity in the study as multitasking compared to talking on the phone and answering work emails ( $1=$ Not at all multitasking compared to the example, $4=$ Somewhat multitasking compared to the example, $7=$ Totally multitasking compared to the example), and (iii) whether they would feel like they were completing two different tasks, a single task with two components, or a single task.

$67.4 \%$ of participants who were assigned to the multitasking condition indicated that they perceived they were multitasking relative to $40.4 \%$ of those assigned to the single-tasking $\left(\right.$ Pearson- $\left.\chi_{(1, \mathrm{~N}=178)}^{2}=13.02, p<.001\right)$. The two 7-point scales were highly correlated $(r=.73, p$ $<.001)$ and thus were combined. An ANOVA verified that the manipulation worked as intended $\left(M_{\text {multitasking }}=4.58, \mathrm{SD}=1.49 ; M_{\text {single-tasking }}=3.66, \mathrm{SD}=1.74, F(1,176)=14.51, p<.001\right)$. Additionally, of the participants who were assigned to the multitasking [single-tasking] condition, 37\% [19\%] indicated perceiving the described activity as working on two separate tasks $\left(\right.$ Pearson- $\left.\chi^{2}(1, \mathrm{~N}=178)=7.12, p=.008\right)$, and $17 \%[38 \%]$ indicated perceiving the activity as

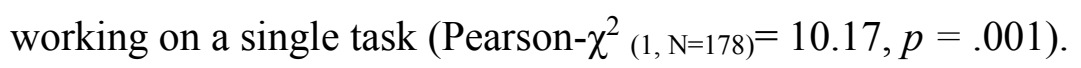

Need for structure. After completing the task, participants navigated to an ostensibly unrelated survey in which they responded to items about their need for structure (Thompson et 
al, 2001). One participant did not respond to the need for structure items and was therefore eliminated from the analysis. Thus, for the remaining one hundred and sixty-one participants, we averaged and mean-centered their responses on the need for structure scale. We tested whether the effect of the task framing on the number of words transcribed was moderated by participants' need for structure. A regression analysis revealed that, the main effect of the framing manipulation on the number of words typed was positive and significant $(\beta=25.43, t=$ $2.46, p=.015)$, replicating the proposed effect. Interestingly, the main effect of need for structure on number of words typed was positive and reached marginal significance $(\beta=27.63, t$ $=1.90, p=.059)$, suggesting that, generally, people with a higher need for structure performed better on this specific task. However, we did not find that the need for structure moderated the effect of framing on performance as the interaction was not significant $(\beta=14.45, t=0.99, p$ $>.25)$

\section{Study 1b}

Manipulation Checks. In a separate pretest, using the same population from a northeastern university, we validated the manipulation that was employed. In the pretest, two hundred and seven participants were randomly assigned to read the instructions of the multitasking or single-tasking conditions. Participants were then told that they would be matched with a partner. If their partner and they answered the question in the same way, they would be entered into a lottery for $\$ 50$. The question asked participants to identify whether they would perceive the described activity as a single task activity or a multitask activity. Further, participants indicated to what extent they would feel they were multitasking $(1=$ Not at all, $4=$ 
Somewhat, $7=$ Totally) and when taking this survey whether they would feel like they were completing two different tasks, a single task with two components, or a single task.

Of the participants who were assigned to the multitasking condition, $87.4 \%$ indicated that they would perceive they were multitasking relative to $66.3 \%$ of those assigned to the singletasking condition (Pearson- $\left.\chi^{2}(1, \mathrm{~N}=207)=12.85, p<.001\right)$. An ANOVA verified that participants who were assigned to the multitasking condition indicated that they would perceive they were multitasking more relative to those assigned to the single-tasking condition $\left(M_{\text {multitasking }}=5.28\right.$, $\left.S D=1.41 ; M_{\text {single-tasking }}=4.07, S D=1.79 ; F(1,205)=29.44, p<.001\right)$.

Furthermore, of the participants who were assigned to the multitasking [single-tasking] condition, $17 \%[14 \%]$ indicated perceiving the activity described as working on two separate tasks (Pearson- $\left.\chi^{2}(1, \mathrm{~N}=207)=0.17, p>.25\right)$, and $14 \%[24 \%]$ indicated perceiving the activity as working on a single task Pearson- $\left.\chi^{2}(1, \mathrm{~N}=207)=3.69, p=.055\right)$. Thus, although the last measure did not reach statistical significance, the other two strongly validate that the manipulation worked as intended.

\section{Study 2a}

\section{Additional Information Regarding Method and Procedures}

After all participants finished working on the task we measured the extent to which they perceived their activity as either multitasking or single-tasking. Specifically, participants were given four sets of two scenarios and were asked to indicate, in each set, which of the two scenarios they perceived to be more similar to the activity they had just completed. We framed each scenario to be perceived as either multitasking or single-tasking, but kept the actual activity fixed. The four scenarios were buying a knife set for your father and responding to emails, 
preparing a proposal and presentation for a marketing campaign, filling out a survey and listening to music, and, lastly, balancing corporate books and writing a memo.

As an additional way to measure how participants construed their activity on the task we told participants that they would be matched with a partner, and that if their partner and they both responded to a question in the same way, they would each receive an additional $\$ 0.05$ bonus. The question was whether they perceived working on the tasks as a multitasking or single-task activity.

\section{Study 2b}

\section{Additional Information Regarding Method and Procedures}

As indicated in the main text of the manuscript, since the framing manipulation was relatively subtle, we also included in this study an additional factor intended to further strengthen the manipulation. In particular, we also manipulated (between-subjects) whether or not we disclosed to participants that in this study some participants would be asked to work on a single study while others would be asked to work on two studies in the same amount of time. Thus, potentially making the multitasking manipulation stronger for participants assigned to the disclosure condition. Participants in the no-disclosure condition were not aware of this randomization procedure. The disclosure manipulation did not produce any main effects or interactions on any of the dependent variables or with the framing manipulation. Therefore, we collapsed the results and do not discuss this factor further. Note that doing so does not substantially change the pattern of results.

For exploratory purposes, upon finishing their assignment, participants in all conditions were asked to indicate whether they would prefer to multitask or single-task on a subsequent 
task. Further, we asked participants to indicate (i) their satisfaction with their performance, (ii) how well they believed they performed relative to others, (iii) how productive they felt, (iv) how long they considered the duration of the assignment to be, (v) how quickly they felt time passed, and (vi) how rushed they felt while working (all measured on a 1 to 7 scale).

Participants also responded to several exploratory individual difference questions to understand their attitudes towards multitasking. Specifically, participants indicated the extent to which, (i) they multitasked often, (ii) multitasking helped them be more efficient, (iii) they made more mistakes when multitasking, (iv) multitasking helped them get things done more quickly when they were busy, (v) multitasking helped them do the work at hand, (vi) multitasking was enjoyable, and (vii) multitasking was stressful (all measured on a 1-7 scale). None of these individual difference measures moderated the effect or were influenced by the manipulations; therefore, we do not discuss these further.

\section{Results}

Performance Controlling for Time. The observed increase in performance may be driven by two main factors. Specifically, it is possible that the multitasking manipulation increased performance because participants in this condition worked longer; hence, they found more words. However, it is also possible that aside from working longer, participants in the multitasking condition were more efficient with their time. In order to examine how each of these two sources improved performance, we examined the amount of words identified across the conditions controlling for time (i.e., using an ANCOVA with log transformation of time as a covariate). As expected, we find a main effect of time on performance $(F(1,234)=20.34, p$ $<.001$ ), indicating that the longer participants worked on the tasks the better they performed. Importantly, even after controlling for time, we still found a significant effect of condition on 
performance $(F(1,234)=46.49, p<.001)$, suggesting that participants in the multitasking condition not only worked longer, but also were more efficient in their work.

Additional Analyses. The three questions of subjective performance were combined into a single scale $(\alpha=.92)$. An ANOVA revealed that participants assigned to the multitasking condition believed they performed better compared to those assigned to the single-tasking condition $\left(M_{\text {multitasking }}=3.91, \mathrm{SD}=1.47 ; M_{\text {single-tasking }}=3.50, \mathrm{SD}=1.39, F(1,235)=5.02, p\right.$ $=.026)$. This suggests that, in general, participants' perceptions were calibrated with their actual performance. Therefore, it is unsurprising that participants in the multitasking condition were also more likely to choose to multitask on a subsequent task $(37.50 \%)$ compared with those assigned to the single-tasking condition $\left(23.93 \%\right.$, Pearson- $\left.\chi^{2}(1, \mathrm{~N}=237)=5.12, p=.017\right)$.

Participants assigned to the multitasking condition were marginally more likely to perceive that time passed more quickly than those who experienced the single-tasking condition $\left(M_{\text {multitasking }}=4.86, \mathrm{SD}=1.23 ; M_{\text {single-tasking }}=4.62, \mathrm{SD}=1.03 ; F(1,235)=2.80, p=.096\right)$, and they also felt more rushed $\left(M_{\text {multitasking }}=5.11, \mathrm{SD}=1.58 ; M_{\text {single-tasking }}=4.44, \mathrm{SD}=1.67 ; F(1\right.$, 235) $=9.89, p=.002)$.

\section{Discussion}

This study demonstrates that, holding the actual tasks constant, the mere perception of multitasking improves performance. Participants that construed their activity as multitasking (as opposed to single-tasking) persisted longer and worked more efficiently. Interestingly, participants that perceived their activity as multitasking also reported feeling time had passed more quickly. Such distortions in time perception have been shown in previous research to arise, among other things, from greater engagement and feelings of excitement (Gable \& Poole, 2012). 
Thus, the results may indicate that at least part of the underlying mechanism for the improved performance is due to increased engagement with the task.

\section{Study 3}

\section{Additional Information Regarding Method and Procedures}

One hundred and fifteen participants were recruited from a behavioral lab at a northeastern university $(60 \%$ female, mean age $=20.46)$. At the beginning of the study, we determined participants' dominant eye ${ }^{7}$. Participants were then asked to complete an incentivecompatible assignment identical to that employed and described in Study $2 \mathrm{~b}$ and following the same procedure. Specifically, participants worked on the puzzles for as long as they liked and their pupil dilation and eye movement were tracked using SMI RED-m eye-tracking equipment.

\section{Results}

Persistence. Since the data was censored at four minutes, we used a cox regression survival analysis to compare persistence on the puzzles task across conditions and found that most participants worked for the full 4 minutes (potentially as a result of being directly observed by a research assistant who was monitoring the eye-tracking equipment). Thus, the framing manipulation had no significant effect on how long participants worked on the task (Wald- $\chi^{2}(1$, $\mathrm{N}=107)=0.66, p>.40)$.

Pupil Dilation. Common practices when employing pupil dilation measures are to analyze average pupil dilation (Beatty \& Lucero-Wagoner, 2000) ${ }^{8}$. To examine whether the perception of multitasking increased participants' pupil dilation and to make sure our results

\footnotetext{
${ }^{7}$ If the test was inconclusive (i.e. participants were dominant in both eyes), we coded their dominant eye as their right eye. This coding affected five participants.

${ }^{8}$ Some papers also report maximum and median pupil dilation. Although these measures are typically noisier, we report these as well.
} 
were robust, we analyzed all average, median and maximum pupil dilation measures of participants' dominant eye and used ANOVA analyses on each measure. Participants' average pupil dilation (averaged across the entire duration of the task) was found to be significantly larger in the multitasking condition $(M=3.90, \mathrm{SD}=0.51)$ than the single-tasking condition $(M=$ 3.64, $\mathrm{SD}=0.49 ; F(1,105)=7.12, p=.009)$. The same significant difference was found when analyzing median pupil dilation $\left(M_{\text {multitasking }}=3.81, \mathrm{SD}=0.71, M_{\text {single-tasking }}=3.53, \mathrm{SD}=0.81\right.$; $F(1,105)=3.50, p=.064)$ and maximum pupil dilation $\left(M_{\text {multitasking }}=4.73, \mathrm{SD}=0.65, M_{\text {single- }}\right.$ tasking $=4.47, \mathrm{SD}=0.54 ; F(1,105)=4.89, p=.029)$.

Switches. Using the eye-tracking paradigm, we are able to track participants'switching patterns when working on the tasks. One important concern is whether or not the observed improvement in performance is triggered by a specific work sequence. Although Study 7 will address this concern, in this study we conducted additional analyses to address it further.

The eye-tracking equipment measures participants' pupil dilation and where they are looking on the screen every $60 \mathrm{~Hz}$ (around 16.6 milliseconds) ${ }^{9}$. By defining, a priori, two specific areas of interest on the screen (i.e., the areas in which the word puzzle and anagram appear) we are able to determine which task participants attended to at any given point in time. Furthermore, this allowed us to observe when participants switched between tasks. However, a certain assumption should be made with regards to what constitutes a "switch". In particular, one needs to define the minimum amount of time a participant fixates on a specific task prior to fixating on the other task to be considered a real switch in attention (as opposed to noise or momentary fixation which does not indicate a shift in attention and a task switch). Given the nature of the task, we defined the minimum amount of fixation-time to be one second. That is,

\footnotetext{
${ }^{9}$ Five participants were excluded from the switch analyses because the eye-tracking equipment failed to record their fixation location.
} 
any fixation of less than one second will not be considered as an actual and deliberate switch from one task to the other. Further, since what constitutes a switch is not standardized in previous literature and highly depends on the specific nature of the task, we conducted auxiliary analyses and robustness checks. We find that the results hold for other specifications of a "switch" in which we defined the minimum fixation-time to be two or three seconds.

Pupil dilation controlling for number of switches. One account for why participants' pupils were more dilated in the multitasking condition is that these participants' switched more. Indeed, and unsurprisingly, participants in the multitasking condition switched more $(M=11.83$, $\mathrm{SD}=6.40)$ than those in the single-tasking condition $(M=6.62, \mathrm{SD}=7.39 ; F(1,100)=14.44, p$ $<.001)$. Accordingly, it is possible that merely switching between tasks might have heightened participants' arousal level, and therefore, their dilation. In order to test whether the number of switches drove the increase in pupil dilation, we conducted an ANCOVA in which we controlled for the number of switches (defined as at least one-second fixation on one task followed by at least one-second fixation on the other task). We found that even after controlling for the number of switches, participants' average pupil dilation was higher in the multitasking condition $(F(1$, $99)=5.90, p=.017)$. This result held also when analyzing participants' median pupil dilation $(F(1,99)=3.26, p=.074)$, and maximum pupil dilation $(F(1,99)=3.85, p=.052)$. Further, the same pattern was observed when we defined switches as two and three second fixations ${ }^{10}$.

Mediation analysis. Next we conducted a mediation analysis (using model 4 of the macro PROCESS; Hayes, 2013) in order to test whether the effect of task framing on performance was mediated by average pupil dilation. The dependent variable was the number of words found, the independent variable was task framing (multi vs. single-tasking), and the mediator was

${ }^{10}$ Excluding maximum pupil dilation, which was a much noisier measure. 
participants' average pupil dilation. The indirect effect was tested using a bootstrap estimation approach with 5000 samples and was found to be significant $(\beta=0.59, \mathrm{SE}=.34,95 \% \mathrm{CI}$ $=.1008,1.5524)$. Thus, as hypothesized, the effect of task framing on performance was mediated by participants' pupil dilation. The mediation results hold when analyzing median or maximum pupil dilation.

Pupil dilation for a fixed number of words found. Given the correlational nature of any mediation analysis, one should be cautious with causality interpretations. For example, although manipulating the perception of multitasking increased performance (i.e., participants found more words), it is possible that the increase in pupil-dilation was not the cause for the increase in performance but rather its outcome. In particular, it is possible that because participants found more words in the multitasking condition their pupils dilated due to happiness and excitement. While we cannot fully rule out this account, an additional analysis that controls for the number of words found in each condition casts doubt on this interpretation. Specifically, we first examined participants' average pupil dilation up until they found their first word in the puzzles. Again, participants' pupil dilation was greater in the multitasking than the single-tasking condition $\left(M_{\text {multitasking }}=3.93, \mathrm{SD}=0.52, M_{\text {single-tasking }}=3.71, \mathrm{SD}=0.57 ; F(1,105)=4.28, p=.041\right)$. The results hold when we look at participants' pupil dilation up until they identified their second, third, fourth, and fifth word (all $p$ 's $<.08$ ). Thus, the results cast doubt on the argument the participants' pupils dilated more in the multitasking condition just because they found more words.

\section{Discussion}

In this study, we first show that the perception of multitasking increases pupil dilation, which mediates performance. Thus, this study provides physiological evidence that the mere 
perception of multitasking (holding the activity constant) increases engagement with, and performance on the task.

\section{Study 4 - Sense of Agency}

Oftentimes in life, individuals have the freedom to choose whether or not to multitask. Thus, to explore the robustness and ecological validity of the findings, this study aimed to test whether or not the observed effect is limited to situations in which individuals' activity of multitasking or single-tasking is externally imposed. Therefore, in this study we also manipulated participants' sense of agency in deciding whether or not to multitask.

\section{Method}

One hundred and fifty-nine paid online participants were recruited from Amazon.com's Mechanical Turk $(48 \%$ female; mean age $=31.61)$. The procedure and methods were identical to those described in Study 2b. In addition to manipulating the task framing (multitasking vs. single-tasking), we also manipulated whether participants had a sense of agency over the decision of whether or not to multitask. Thus, the study was a 2 (multitasking condition vs. single-tasking condition) $\times 2$ (agency vs. no agency) between-subjects design. In the no agency condition, participants were randomly assigned to either the multitasking or single-tasking condition. In contrast, in the agency condition, we gave participants a choice of whether to work on an assignment that would require them to multitask or single-task. In order to prevent a selfselection bias, we randomly assigned participants in the agency condition to one of two incentive schemes. In one scheme, participants were informed that the average earnings of the multitasking assignment were high $(\$ 0.13)$ compared to the single task assignment $(\$ 0.02)$. This encouraged participants in this scheme to choose the multitasking assignment, yet maintained a sense of 
agency in the form of free choice. In the second incentive scheme, we told participants that the average earnings of the single task assignment were higher (\$0.13) compared to the multitasking assignment (\$0.02). In order to keep the incentive scheme as similar as possible across all conditions, participants in the no agency condition were also told that the average pay for the assignment they engaged in (either multitasking or single-tasking) was $\$ 0.13$.

The majority of participants (97\%) chose the assignment with higher expected earnings; thus, almost completely eliminating the possibility that a self-selection bias would significantly impact our results. However, in order to be as conservative as possible, in an auxiliary analysis, the 5 participants that chose to work on the lower-earning tasks were coded in a way that counters our hypothesis. Even after conducting this extremely conservative test, all results reported below hold.

As a manipulation check, participants indicated to what extent they were multitasking $(1=$ Not at all, 4= Somewhat, $7=$ Totally) and whether they felt like they were completing two different tasks, a single task with two components, or a single task (selected one of the three descriptions that matched their experience).

For exploratory purposes, upon finishing their assignment, participants in all conditions were asked to respond to the same post-task questions detailed in Study $2 \mathrm{~b}$ (i.e., items relating to subjective feelings of performance, willingness to multitask on a subsequent task, perception of time, and attitudes). In addition, participants also indicated (i) how difficult they found the assignment to be (using two items), (ii) how efficiently they used their time while working on the assignment, and (iii) the extent they found the activity to be enjoyable, and (iv) the extent they found the activity to be challenging (all measured on a 1 to 7 scale).

\section{Results}


Manipulation Checks. An ANOVA verified that the manipulation had the intended effect. Participants assigned to the multitasking condition indicated that they perceived their activity as multitasking to a greater extent than those assigned to the single-tasking condition $\left(M_{\text {multitasking }}=\right.$ $\left.4.76, \mathrm{SD}=1.64 ; M_{\text {single-tasking }}=2.46, \mathrm{SD}=1.64 ; F(1,150)=75.59, p<.001\right)$. As expected there was no impact of agency $(F(1,150)=0.10, p>.70)$ or an interaction between the agency and task framing manipulations on the extent to which participants indicated they were multitasking $(F(1,150)=0.73, p>.39)$. Furthermore, of the participants who were assigned to the multitasking [single-tasking] condition, 34\% [8\%] indicated perceiving their activity as working on two separate tasks (Pearson- $\left.\chi^{2}(1, \mathrm{~N}=154)=15.01, p<.001\right)$, and $4 \%[69 \%]$ indicated perceiving their activity as working on a single task (Pearson- $\left.\chi^{2}(1, \mathrm{~N}=154)=71.70, p<.001\right)$. Thus, the framing manipulation worked as intended.

Performance. An ANOVA revealed that participants assigned to the multitasking condition submitted, on average, more words $\left(M_{\text {multitasking }}=14.31, \mathrm{SD}=6.91\right)$, than those assigned to the single-tasking condition $\left(M_{\text {single-tasking }}=8.22, \mathrm{SD}=4.53 ; F(1,150)=42.97, p\right.$ $<.001)$. The analysis also revealed a main effect of agency on performance $\left(M_{\text {agency }}=12.63, S D\right.$ $\left.=6.83 ; M_{\text {no_agency }}=10.26, S D=6.24 ; F(1,150)=5.25, p=.023\right)$. However, more pertinent to the goal of this study, the interaction between agency and framing manipulation did not reach statistical significance $(F(1,150)=2.60, p=.109)$ indicating that participants perceiving their activity as multitasking, performed better regardless of whether or not they had a sense of agency over the decision to multitask.

Persistence. Participants assigned to the multitasking condition spent, on average, longer on the task $\left(M_{\text {multitasking }}=2.35, \mathrm{SD}=0.09\right)$ compared to those assigned to the single-tasking condition $\left(M_{\text {single-tasking }}=2.25, \mathrm{SD}=0.31\right)$. However, the results failed to reach statistical 
significance $\left(\right.$ Wald $\left.-\chi_{(1, \mathrm{~N}=154)}^{2}=0.13, p>.70\right)$. No main effects of the agency manipulation or its interaction with the framing manipulation were observed (Wald- $\chi^{2}(1, \mathrm{~N}=154)=0.41, p>.52$;

Wald- $\chi_{(1, \mathrm{~N}=154)}^{2}=2.59, p=.108$, respectively). In all subsequent analyses, no other main effects of the agency manipulation nor its interaction with the framing manipulation reached statistical significance. Thus, we collapsed the results across this factor and do not discuss it further.

Additional Analyses. The three questions of subjective performance were combined into a single scale $(\alpha=.91)$. An ANOVA revealed that participants assigned to the multitasking condition believed they performed better compared to those assigned to the single-tasking condition $\left(M_{\text {multitasking }}=4.10, \mathrm{SD}=1.40 ; M_{\text {single-tasking }}=3.58, \mathrm{SD}=1.39 ; F(1,152)=5.52, p\right.$ $=.020)$. Suggesting that, in general, participants' perceptions were calibrated with their actual performance. Replicating the observed pattern, participants assigned to the multitasking condition were more likely to choose to multitask on a subsequent task (57.50\%) compared with those assigned to the single-tasking condition $\left(16.22 \%\right.$; Pearson- $\left.\chi^{2}(1, \mathrm{~N}=154)=27.91, p<.001\right)$.

Participants assigned to the multitasking condition indicated feeling that time passed more quickly than those assigned to the single-tasking condition $\left(M_{\text {multitasking }}=5.19, \mathrm{SD}=1.41\right.$; $\left.M_{\text {single-tasking }}=4.69, \mathrm{SD}=1.83 ; F(1,152)=3.63, p=.059\right)$, and also felt more rushed $\left(M_{\text {multitasking }}\right.$ $\left.=4.81, \mathrm{SD}=1.48 ; M_{\text {single-tasking }}=4.24, \mathrm{SD}=1.93 ; F(1,152)=4.25, p=.041\right)$. All other measures (i.e., perception of task difficulty, estimation of minutes spent on activity, enjoyment, and perceived efficiency) were all not affected by the framing manipulation (all $p$ 's $>.25$ ).

\section{Discussion}

This study demonstrates that the mere perception of multitasking improves performance regardless of whether or not individuals have a sense of agency over the decision to multitask or not. 


\section{Study 5 - Extraversion and Neuroticism}

Previous research has demonstrated that individuals' tendency to habituate and adapt impacts their task performance (Deutsch \& Deutsch, 1963). In particular, when people habituate to an activity they pay less attention to it, which could hurt performance on that activity. Accordingly, one potential reason for the observed difference in performance across the framing conditions is that individuals who perceive their activity as multitasking habituate slower and therefore perform better. If such a mechanism is the main driver of the effect, one should expect to find that the impact of the framing manipulation on performance should be stronger for individuals who tend to adapt quicker to activities. Building on previous literature, in this study we test this habituation account. In particular, previous literature found that individuals who are typically quicker to adapt to different stimuli and environments, score high on extraversion and neuroticism scales (Mangan \& O'Gorman, 1969). Thus, to the extent that rate of habituation is a driver of the effect, one should expect to see that the effect would be stronger for people who score higher on these scales. To test this, in the current study we measured participants' individual differences on the extraversion and neuroticism scales and examined whether these would moderate the observed effect.

\section{Method}

Six hundred six paid online participants were recruited from Amazon.com's Mechanical Turk $(48 \%$ female; mean age $=36.09)$. The procedure and methods were identical to those described in Study 2b. As a proxy for participants' tendency to adapt to different stimuli, participants also completed the extraversion and neuroticism sub scales of Eysenck's Personality 
inventory (Eysenck \& Eysenck, 1975) either before or after the main tasks in this study (manipulated between-subjects).

\section{Results}

Whether participants answered the neuroticism and extraversion questions before or after working on the task did not significantly impact the pattern of results and thus we present the results collapsed across this factor.

Performance. An ANOVA showed that participants in the multitasking condition performed better $(M=12.50, \mathrm{SD}=7.90)$ than participants in the single-tasking condition $(M=$ $8.35, \mathrm{SD}=6.38 ; F(604)=50.43, p<.001)$.

Persistence. A cox regression survival analysis revealed that participants assigned to the multitasking condition worked longer on the task $(M=2.34, \mathrm{SD}=0.13)$ compared to participants in the single-tasking condition $\left(M=2.25, \mathrm{SD}=0.32\right.$; Wald- $\left.\chi_{(1, \mathrm{~N}=606)}^{2}=13.27, p<.001\right)$.

Extraversion and Neuroticism. Participants' responses to the extraversion scale were averaged and mean-centered. A regression analysis revealed that although the main effect of the framing manipulation was significant $(\beta=2.09, t=7.15, p<.001)$, neither participants' extraversion nor its interaction with the framing manipulation was statistically significant $(\beta=-$ $0.07, t=-1.45, p=.147 ; \beta=0.00, t=0.08, p>.93)$. A similar procedure was employed for examining participants' responses to the neuroticism scale. Again, neither neuroticism nor its interaction with the framing manipulation significantly impacted performance $(\beta=-0.04, t=$ $0.85, p>.39 ; \beta=-0.01, t=-0.32, p>.74$, respectively).

\section{Discussion}


By measuring participants' neuroticism and extraversion, we find no evidence to support that the effect of the framing manipulation on performance is driven by individuals' tendency to adapt more slowly to different stimuli.

\section{Study 6 - Work Related Constructs}

The goal of this study was to test whether framing an activity as multitasking will impact work related constructs. Specifically, previous research demonstrated that individuals' selfefficacy and locus of control are positively correlated with performance (Judge \& Bono, 2001; Stajkovic \& Luthans, 1998). Therefore, in the current study, after administering the manipulation, we measured Rotter's locus of control scale (Rotter, 2011) or Sherer et al.'s selfefficacy scale (Sherer et al, 1982) to test whether these were affected by the task framing.

\section{Methods}

One hundred and eighty-three participants from Amazon's Mechanical Turk (44\% female; mean age $=37.96$ ) were recruited to take part of a study. Participants were assigned to either a multitasking or single-tasking condition (between-subjects) and the descriptions of the tasks in this study were identical to those in Study 2b. Participants were led to believe that they were going to work on the tasks but prior to beginning were asked to respond to several items. In particular, we randomly assigned participants to respond to either a 9-item version of Rotter's locus of control scale (Rotter 2011) or Sherer et al.'s self-efficacy scale (Sherer et al, 1982). This randomization procedure ensured that participants' responses to one scale would not affect their responses to the other. Consequently, participants responded to either all of the items from the self-efficacy scale or the locus of control scale (items for both scales are below). 
Items used to measure locus of control:

1. My life is determined by my own actions.

2. I am usually able to protect my personal interests.

3. I can pretty much determine what will happen in my life.

4. To a great extent, my life is controlled by accidental happenings. (R)

5. Often there is no chance of protecting my personal interest from bad luck happenings. (R)

6. When I get what I want, it's usually because I'm lucky. (R)

7. People like myself have very little chance of protecting our personal interests where they conflict with those of strong pressure groups. (R)

8. My life is chiefly controlled by powerful others. (R)

9. I feel like what happens in my life is mostly determined by powerful people. (R)

Items used to measure self-efficacy:

1. When I make plans, I am certain I can make them work.

2. One of my problems is that I cannot get down to work when I should. (R)

3. If I can't do a job the first time. I keep trying until I can.

4. When I set important goals for myself, I rarely achieve them. (R)

5. I give up on things before completing them. (R)

6. I avoid facing difficulties. (R)

7. If something looks too complicated, I will not even bother to try it. (R)

8. When I have something unpleasant to do, I stick to it until I finish it.

9. When I decide to do something, I go right to work on it. 
10. When trying to learn something new, I soon give up if I am not initially successful.

11. When unexpected problems occur, I don't handle them well. (R)

12. I avoid trying to learn new things when they look too difficult for me. (R)

13. Failure just makes me try harder.

14. I feel insecure about my ability to do things. (R)

15. I am a self-reliant person.

16. I give up easily. (R)

17. I do not seem capable of dealing with most problems that come up in life. (R)

\section{Results}

Locus of control. $(\mathrm{N}=89)$ Participants' responses to the Locus of Control scale were averaged $(\alpha=.59)$. An ANOVA showed that the task framing manipulation did not affect participants' locus of control $\left(M_{\text {multitasking }}=4.51, \mathrm{SD}=1.05 ; M_{\text {single-tasking }}=4.86, \mathrm{SD}=1.07 ; F(1\right.$, 87) $=2.36, p=.128)$.

Self-efficacy. (N=94) Participants' responses to the self-efficacy scale were averaged ( $\alpha$ $=.67$ ). The results of an ANOVA showed that the task framing manipulation did not affect participants' self-efficacy $\left(M_{\text {multitasking }}=5.32, \mathrm{SD}=1.13, M_{\text {single-tasking }}=5.20, \mathrm{SD}=0.87 ; F(1,92)\right.$ $=0.34, p>.56)$.

\section{Discussion}

In this study, we find no evidence to support that the task framing impacted the work related constructs, Locus of Control and self-efficacy. Thus, we find no evidence to support that the effect of the framing manipulation on performance is driven by a shift in individuals' Locus of Control or self-efficacy. 


\section{Study 7 - Switching Pattern}

In the studies reported thus far, we find that holding constant the actual task, the mere perception of multitasking improved performance. However, while we controlled for the actual task, in Studies 4-6 we did not control for the sequence in which participants performed the task. In particular, participants were free to switch whenever they wanted between the word puzzle and anagram tasks. It is possible, for example, that the framing manipulation merely changed the work sequence (i.e., participants switched sooner versus later, or switch more versus less frequently, etc.). Although, this may be part of what is driving the proposed effect, it does raise a certain concern. Specifically, one could argue that the findings are limited to specific tasks that favor one sequence over the other. In accordance with this concern, one may argue that for certain tasks that benefit from a different work sequence; the perception of multitasking would actually impede performance.

We address this concern in several distinct ways throughout the paper using our experimental designs and analyses (Studies 1a, 1b and 3). In this study, we address this concern by directly controlling the work sequence that participants use. In particular, instead of allowing participants to switch between tasks whenever they like, in this study we externally impose a switching pattern. Because restricting the pattern in which participants switch back and forth might reduce their sense of agency, we also manipulated participants' sense of agency over the switching pattern to address this concern.

\section{Method}

One hundred and sixty-one paid online participants $(52 \%$ female; mean age $=36.99)$ were recruited from Amazon.com's Mechanical Turk and were asked to complete an incentive- 
compatible assignment. The assignment was comprised of two tasks. The first task was a word location task in which participants were given a pair of numbers to identify a word in different passages taken from Shakespeare's Hamlet. In particular, the first number indicated the row in the text that the word was located in, and the second number indicated its location in that specific row. For example, the number pair 14-8 indicated that the word participants needed to identify was the $8^{\text {th }}$ word on the $14^{\text {th }}$ line.

The second task was a letter-count task. In this task, participants were given different passages taken from Mary Shelley's Frankenstein and asked to count the number of times a specific letter appeared in that passage. For both tasks, participants were asked to choose the right answer (either word or number depending on the specific task) from five multiple-choice options. There were a total of 24 questions and, for each correct answer participants earned an additional $\$ 0.02$.

Although the tasks were held constant across conditions, we randomly assigned participants to either the multitasking or single-tasking condition. Participants assigned to the multitasking condition learned that they would work on two distinct studies (labeled the "Cognitive Study" and the "Visual Study") comprised of the aforementioned word location and letter-count tasks respectively. In order to make it salient that these were two different studies, we employed different fonts and background colors for each task. In the single-tasking condition, the two tasks were framed as part of a single study (labeled the "Cognitive-Visual Study") comprised of two types of questions. The instructions for the tasks were identical in both conditions and, although participants were told that they should finish the task in 9 minutes, they were actually allowed to work on the task for as long as they liked. 
Unlike the Studies 4-6, in which both tasks were simultaneously visible on the screen, in this study participants observed only a single type of task at any point of time (either the wordlocation or the letter count task). Further, the task type alternated after every two questions. Thus we externally controlled the work sequence and switching pattern (i.e., the task type switched every two questions).

Because imposing a strict switching pattern may reduce participants' sense of agency, we also manipulated (between-subjects) participants' sense of agency. In particular, in the agency condition, participants were asked to choose, in advance, the frequency with which they would switch from one type of question to the other (every 2, 4, or 6 questions). Participants assigned to the no agency condition were not given this choice and they had to switch every two questions. In order to keep the experimental conditions as similar as possible, we motivated participants in the agency condition to choose to switch every two questions by telling them that based on the performance of other participants taking this study in the past, the most recommended frequency of switching was every two questions. Thus, the agency manipulation aimed to give participants the illusion of agency while keeping the switching patterns as similar as possible to those in the no agency manipulation. Indeed, the vast majority of participants in the agency condition (95\%) chose to switch every two questions (analyzing the data with or without the 8 participants, 4 in the multitasking and 4 in the single-tasking condition, that chose a different switching pattern does not substantially change the pattern of results).

As measures of engagement, once participants finished working on the tasks, they reported how tired they felt, and how bored they were (both measured on a 1 to 7 scale). As a manipulation check for the task framing, participants indicated to what extent they were multitasking $(1=$ Not at all, $4=$ Somewhat, $7=$ Totally $)$ and whether they felt like they were 
completing two different tasks, a single task with two components, or a single task (selected one of the three descriptions that matched their experience). As a manipulation check for sense of agency, participants indicated to what extent they felt they had control over the switching pattern between the two studies.

For exploratory purposes, upon finishing their assignment, participants in all conditions were asked several items adapted from Keinan (2007)'s productivity mindset measures. Further, we asked participants to indicate their satisfaction with their performance on the task, how productive they felt, and their level of distraction during the task, (all measured on a 1 to 7 scale). Finally, participants responded to Sherer et al.'s (2008) self-efficacy scale to again test whether this was affected by the task framing manipulation.

\section{Results}

Framing Manipulation Checks. Using an ANOVA we examined whether the manipulation worked as intended. Participants who were assigned to the multitasking condition indicated that they perceived that they were multitasking more relative to those assigned to the single-tasking condition $\left(M_{\text {multitasking }}=3.92, S D=1.83 ; M_{\text {single-tasking }}=3.09, S D=1.66 ; F(1,151)=\right.$ $8.65, p=.004)$. Of the participants who were assigned to the multitasking [single-tasking] condition, 30\% [26\%] indicated perceiving their activity as working on two separate tasks $\left(\right.$ Pearson- $\left.\chi_{(1, \mathrm{~N}=153)}^{2}=0.30, p>.58\right)$, and $6 \%[16 \%]$ indicated perceiving their activity as working on a single task (Pearson- $\left.\chi_{(1, \mathrm{~N}=153)}^{2}=4.02, p=.045\right)$. Admittedly, we can see that the difference in perception, though statistically significant on the continuous measure (which offers more statistical power), is relatively small. This is not surprising given the specific characteristics of how we designed the stimuli in this study (i.e., observing the tasks sequentially 
as opposed to simultaneously). Nevertheless, we can still see a statistically significant shift in the continuous measure and a weaker directional shift in the choice measures.

Agency Manipulation Check. An ANOVA verified that participants assigned to the agency condition reported having more control over the switching pattern of the questions than those assigned to the no agency condition $\left(M_{\text {agency }}=3.82, S D=1.84 ; M_{\text {no_agency }}=2.95, S D=1.82\right.$; $F(1,151)=10.16, p=.002)$. However, because this manipulation did not impact our dependent variables or interact with task framing (with the exception of participants' perceptions of their performance), we collapsed the results and do not discuss this factor further. Note that doing so does not substantially change the pattern of results.

Performance. We next examined the average number of correct answers across conditions. Because there were overall 24 questions and because some respondents completed all questions the data was right censored. Therefore, we analyzed the data using a cox regression survival analysis in which we regressed the number of questions answered correctly on the task framing manipulation. Participants assigned to the multitasking condition answered more questions correctly compared to those assigned to the single-tasking condition $\left(M_{\text {multitasking }}=\right.$ 12.32, $S D=6.24 ; M_{\text {single-tasking }}=9.79, S D=5.75 ;$ Wald $\left.-\chi^{2}(1, \mathrm{~N}=153)=6.80, p=.009\right)$.

Persistence. An ANOVA indicated that participants assigned to the multitasking condition worked, on average, longer on the assignment compared to those assigned to the single-tasking condition $\left(M_{\text {multitasking }}=2.64, S D=0.33 ; M_{\text {single-tasking }}=2.50, S D=0.41 ; F(1,151)=\right.$ 4.92, $p=.029)$.

Boredom. The two items measuring boredom and fatigue were correlated $(r=.54, p$ $<.001)$ and collapsed. Despite working on average 90 seconds longer, participants assigned to the multitasking condition reported being less bored and less tired after working on the tasks 
compared to those assigned to the single-tasking condition $\left(M_{\text {multitasking }}=2.99, S D=1.34 ; M_{\text {single- }}\right.$ tasking $=3.52, S D=1.59 ; F(1,151)=4.89, p=.028)$.

Additional Analyses. There was no evidence to suggest that the task framing manipulation impacted any of the items regarding subjective performance, general feelings of productivity, or distraction (all $p$ 's $>.34$ ). Further, we again find no evidence to suggest that participants' feelings of self-efficacy were impacted by the task framing manipulation $(F(1,151)$ $=2.19, p=.141)$.

\section{Discussion}

Study 7 replicates the reported effect using new tasks and a different paradigm (word location task and letter-count task) and shows that the perception of multitasking increases persistence and improves performance regardless of participants' switching pattern or sense of agency over the switching pattern. Interestingly, participants in the multitasking condition also reported being less bored and tired, despite spending longer on the task. This, again, suggests that participants in the multitasking condition were more engaged and aroused during the activity when such activity was construed as multitasking as opposed to single-tasking.

\section{Meta-analysis}

We conducted a total of 23 studies with 6,768 participants. In order to determine the effect size on performance, we conducted a meta-analysis on all the data we had collected using the paradigms from this paper. For each study, we calculated the Cohen's $d$ for the main effect of task framing (multi- vs. single-tasking) regardless if an additional factor was manipulated or if we required all participants to work the same amount of time. Thus, we calculated a conservative estimate for the effect size. In order to calculate each study's Cohen's d, we subtracted the 
average performance in the multitasking condition by the average performance found in the single-tasking condition and divided the difference by the pooled standard deviation. We then weighted the Cohen's d based on the inverse variance of the study's sample. The results of a test of homogeneity revealed that the variability observed across the effect sizes exceeds what would be expected from sampling error $(\mathrm{Q}(22)=59.13, p<.001)$. Thus, we estimated an average Cohen's $d=.501(95 \%$ Confidence Interval $.416, .586)$ using a random effects model (Macro from Wilson, 2006).

\section{Analyzing Performance as a Function of Perceptions}

In 12 of the 23 studies that we conducted, we directly measured participants' perception of multitasking. Across these 12 studies with 3,527 participants, we pooled individuals' performance and the perception of multitasking. These 12 studies employed either the first manipulation check item listed below or both which we used to measure the perception of multitasking ${ }^{11}$.

Item 1: To what extent did you feel like you are multitasking on these studies $(1=$ Not at all, $4=$ Somewhat, $7=$ Totally)?

Item 2: Multitasking is the handling of more than one task as the same time. For example, if Joe was watching a movie while responding to work emails on his iPhone, he would be multitasking. To what extent do you perceive the activity you worked on as multitasking compared to the example about Joe $(1=$ Not at all multitasking compared to the example,

${ }^{11}$ If both items were employed we averaged the two together as they were highly correlated. 
4= Somewhat multitasking compared to the example, 7 = Totally multitasking compared to the example)?

We then normalized the performance data using z-scores and regressed the normalized (also using z-scores) perception of multitasking on performance. As hypothesized, we find a small but significant positive relationship between measured perception of multitasking and performance $(\beta=0.029, t=3.67, p<.001)$.

Table S1: Performance medians, means, and standard deviations for each study by condition.

\begin{tabular}{ccccc|ccc} 
& & \multicolumn{3}{c}{ Multitasking } & \multicolumn{3}{c}{ Single-tasking } \\
Study & Measure & Median & Mean & SD & Median & Mean & SD \\
\hline Study 1a & words transcribed & 280.50 & 274.13 & 126.24 & 249.50 & 229.60 & 137.22 \\
Study 1a & pop quiz score & 7.00 & 6.60 & 1.80 & 6.00 & 5.81 & 2.31 \\
Study 1b & quiz score & 22.00 & 21.00 & 5.29 & 19.00 & 18.23 & 5.71 \\
Study 2b & words submitted & 13.50 & 14.42 & 7.53 & 7.00 & 8.08 & 5.03 \\
Study 3 & words submitted & 15.00 & 18.21 & 11.93 & 8.00 & 10.65 & 7.94 \\
Study 4 & words submitted & 14.00 & 14.31 & 6.91 & 9.00 & 8.22 & 4.53 \\
Study 5 & words submitted & 11.00 & 12.50 & 7.90 & 7.00 & 8.35 & 6.38 \\
Study 7 & questions & 12.00 & 12.32 & 6.24 & 10.00 & 9.79 & 5.75 \\
\hline
\end{tabular}




\section{References Referred to in Supplemental Materials}

Beatty J. \& Lucero-Wagoner B. (2000). The Pupillary System. Handbook of Psychophysiology $2,142-62$.

Deutsch .J.A. \& Deutsch D. (1963). Attention: Aome Theoretical Considerations. Psychological Review, 70, 80-90.

Eysenck, H.J. \& Eysenck, S.B.G. (1975). Manual of the Eysenck Personality Questionnaire. Kent, UK: Hodder \& Stoughton.

Hayes, A.F. (2013). Introduction to Mediation, Moderation, and Conditional Process Analysis, New York, NY: Guilford.

Gable, P.A. \& Poole, B.D. (2012). Time Flies When You're Having Approach-motivated Fun: Effects of Motivational Intensity on Time Perception. Psychological Science, 23, 879-86.

Judge, T.A. \& Bono, J.E. (2001). Relationship of Core Self-evaluations Traits—self-esteem, generalized self-efficacy, locus of control, and emotional stability—with Job Satisfaction and Job Performance: A Meta-analysis. Journal of Applied Psychology, 86(1), 80-92.

Keinan, A. (2007). Productivity Mindset and the Consumption of Collectable Experiences. Doctoral dissertation, Columbia University.

Mangan G.L. \& O'Gorman J.G. (1969). Initial Amplitude and Rate of Habituation of Orienting Reaction in Relation to Extraversion and Neuroticism. Journal of Experimental Research in Personality, 3(4), 275-82.

Rotter J. (2011). Internal-External Locus of Control Scale. 28 MEASURES OF LOCUS OF CONTROL, 10 . 
Sherer, M., Maddux J.E., Mercandante, B., Prentice-Dunn, S., Jacobs, B., \& Rogers R.W. (1982). The Self-efficacy Scale: Construction and Validation. Psychological reports 51(2), 663-671.

Stajkovic, A.D. \& Luthans, F. (1998). Self-efficacy and Work-related Performance: A metaanalysis. Psychological Bulletin, 124(2), 240-61.

Thompson, M.M., Naccarato, M.E., Parker, K.C., \& Moskowitz, G.B. (2001). The Personal Need for Structure and Personal Fear of Invalidity Measures: Historical Perspectives, Current Applications, and Future Directions. In Cognitive social psychology: The Princeton symposium on the legacy and future of social cognition, 19-39.

Wilson, D. B. (2006). Meta-analysis macros for SAS, SPSS, and Stata. Available at http://mason.gmu.edu/ dwilsonb/ma.html. Retrieved November 6, 2016. 